\title{
INTERNACIONALIZACIÓN EN LA EDUCACIÓN SUPERIOR; PROCESOS ADMINISTRATIVOS, ACADÉMICOS Y DE FORMACIÓN
}

\author{
Alejandro Javier Rojas Jardel - Universidad Viña del Mar, Chile - Alejandro.rojas@uvm.cl
}

Resumen: La internacionalización de los procesos enseñanza-aprendizaje en las IES ha tomado relevancia creciente en los últimos años, lo que ha implicado un desafío administrativo, de gestión, curricular y metodológico para enfocar el desarrollo institucional hacia el ámbito internacional. En este contexto, el presente estudio comienza con una revisión bibliográfica del panorama mundial y regional sobre la internacionalización en las universidades en relación a su marco conceptual, características demográficas del proceso en américa latina, el caribe y Chile, analizando la implicancia de este en los planes estratégicos de las IES, especialmente en aspectos administrativos y de gestión académica, para luego proponer una línea de desarrollo por áreas que pretende integrar este proceso a nivel de pre y posgrado e investigación, relevando aspectos de formación continua, la formación de alumnos, interculturalidad y alianzas estratégicas.

\section{I-. Concepto de Internacionalización y su relación con el de ciudadanía global.}

La internacionalización es un proceso que responde a la dinámica de interacción que se desarrolla en al mundo globalizado de manera creciente, lo que también incluye la educación superior de acuerdo a un concepto de "ciudadanía global", el cual se declara como parte de un proyecto global de la UNESCO, "El proyecto Educación para la Ciudadanía Global (GCE, por su sigla en inglés) forma parte de la Primera Iniciativa para la Educación Global, emitida por el secretario general de las Naciones Unidas en 2012, en la cual, además de GCE, se incluyen otros dos proyectos: la educación para la paz y la educación para el desarrollo sustentable" (UNESCO, $\left.2013^{1}\right)$.

En este sentido, la internacionalización se enmarca en una dinámica de globalización, que se entiende cómo "el elemento catalizador, mientras que la internacionalización es la respuesta construida por los universitarios frente a los efectos homogeneizadores y desnacionalizadores de la globalización" (Gacel-Ávila, 2003, p. 34²).

En relación al concepto de internacionalización en la educación superior ha presentado variaciones en el tiempo, entre las que cabe mencionar a Rudzki, para quién esta "promueve un proceso de cambio organizacional, de innovación curricular, del perfil internacional de los académicos y del personal administrativo, así como la movilidad de los estudiantes, todo ello con la finalidad de lograr la excelencia en la docencia, la investigación y en todas las actividades que son parte de las funciones universitarias" (Rudzki, 1998 pag. 16³)

También encontramos los aportes de Hans de Wit, quién la define como "el proceso intencional de integrar una dimensión internacional, intercultural y global en los propósitos, funciones y provisión de la educación terciaria, buscando incrementar la calidad de la educación

\footnotetext{
${ }^{1}$ UNESCO (2013). Global Citizenship Education: An Emerging Perspective.

${ }^{2}$ Gacel-Ávila, J. (2003). La internacionalización de la educación superior. Paradigma para la ciudadanía global. Guadalajara: Universidad de Guadalajara.

${ }^{3}$ Rudzki, R. (1998). "The strategic management of internationalization-Towards a model model of theory and practice". Tesis Doctoral, Newcastle, School of Education, University of Newcastle upon Tyne.
} 
y la investigación para todos los estudiantes y el personal de las instituciones, con la finalidad de hacer una contribución significativa a la sociedad" (De Wit, H., 2001). ${ }^{4}$

Otro acercamiento en la definición del concepto lo describe como "un proceso educativo que integra en las funciones sustantivas universitarias una dimensión global, internacional, intercultural, comparada e interdisciplinaria, que pretende fomentar en los estudiantes una perspectiva global de las problemáticas humanas y una conciencia global en pro de los valores y las actitudes de una ciudadanía global responsable, humanista y solidaria”. (Gacel-Ávila, 2006, p. $61)^{5}$.

Un concepto interesante de la internacionalización que integra elementos valóricos, administrativos y académicos, lo encontramos en el de "internacionalización comprehensiva" o integral, la que se entiende como "un compromiso, confirmado a través de la acción, de integrar las perspectivas internacionales y comparativas en la enseñanza, la investigación y los servicios de la IES, el espíritu (ethos) y valores de una institución, modificándola" (Hudzik, 2011). ${ }^{6}$

En este sentido, la internacionalización comprehensiva propone una integración de varios aspectos a considerar, lo que en conjunto va conllevando un cambio cultural, de la institución en su conjunto, de acuerdo a lo planteado por Gacel-Ávila al señalar que "la reconfiguración global de las economías, los sistemas de intercambio, investigación y comunicación, y el impacto de las fuerzas globales en la vida local demandan una internacionalización comprehensiva alineada a los motivos y propósitos que los guían" (Gacel-Ávila, pág. 15, 2017). ${ }^{7}$

En relación a las perspectivas de internacionalización en la educación superior, estas se analizaron en la "Conferencia Regional de Educación Superior de América Latina y el Caribe" CRES 2018, en Córdoba, donde se planteó que "una internacionalización humanista y solidaria contribuye a generar un mayor y mejor entendimiento y cooperación entre las culturas y las naciones, estimulando una colaboración interinstitucional basada en la solidaridad y el respeto mutuo, y generando una situación de ganar-ganar para todos los socios" (CRES, 2018). ${ }^{8}$

Sin embargo, en un estudio de CINDA se plantea "Es importante remarcar que la internacionalización de la educación superior no necesariamente debe estar atada a una liberación del comercio de servicios educativos", ${ }^{9}$ donde juega un papel fundamental la definición que la universidad pueda darse de internacionalización, no como un fenómeno importado y apropiado acríticamente sino como una estrategia pensada e instrumentada desde el contexto local, las características y necesidades de la comunidad en que dicha institución está inserta.

En este sentido, se puede tomar el ejemplo de España, a través del ministerio del deporte, educación y cultura, que señala ante contexto político y económico de la globalización, la educación superior define sus ejes de desarrollos donde la internacionalización "va más allá de la mera movilidad de estudiantes y firma de acuerdos internacionales, incluyendo

\footnotetext{
${ }^{4}$ De Wit, H. (2001). internationalisation of higher education in the United states of America and europe: A historical, comparative and conceptual analysis. Westport: Greenwood.

${ }^{5}$ Gacel-Ávila, J. (2006). la dimensión internacional de las universidades. contexto, procesos, estrategias. Guadalajara, México: Universidad de Guadalajara.

${ }^{6}$ Hudzik, J. K. (2011). comprehensive internationalization: from concept to action. Washington: nafsa.

${ }^{7}$ Gacel-Ávila, Jocelyne. (2017) “Estrategias de internacionalización de la educación superior: implementación, evaluación y rankings, México: UNESCO-IESALC).

${ }^{8}$ CRES 2018 "Conferencia Regional de Educación Superior de América Latina y el Caribe”, Córdova, Argentina.

${ }^{9}$ Desde el sur: miradas sobre la internacionalización. - 1a ed. - Remedios de Escalada: Ediciones de la UNLa Universidad Nacional de Lanús, 2014, Buenos Aires, Argentina.
} 
aspectos de internacionalización de los currículum formativos, circulación de cerebros, internacionalización de la investigación, titulaciones internacionales conjuntas o múltiples con socios extranjeros, internacionalización en casa, desarrollo de campus transnacionales, creación de sistemas internacionales de aseguramiento de la calidad, acreditación y verificación, competición por los mejores alumnos, clasificaciones internacionales (ranking) de universidades, desarrollo de asociaciones de antiguos alumnos ("alumni”) internacionales, intercambio de experiencias y buenas prácticas, empleabilidad y emprendimiento, etc." 10

En relación al proceso de internacionalización comprensiva descrito anteriormente el énfasis se orienta desde lo individual hacia la institución en su conjunto, implicando un cambio cultural más allá de las pasantías individuales, donde la universidad flexibiliza sus límites de identidad, para abrirse e incorporar elementos de cultura país con quien realiza el intercambio.

Estos a su vez son impactados en distintos niveles; micro (Proceso de enseñanzaaprendizaje), mediano (Estructuras y políticas curriculares) y macro (Toma de decisiones y diseño de políticas).

Como parte de este enfoque conceptual de la internacionalización, encontramos la "internacionalización en casa" (Wachter, 2003) ${ }^{11}$, donde "dicha estrategia se ha desarrollado para prestar atención a aspectos de la internacionalización que se presentan dentro de la propia universidad o en casa".

También encontramos autores que reflexionan sobre la "internacionalización del currículum" (Bennell y Pierce, 2003) comúnmente adoptada a través del "infusion approach", que consiste en infundir al currículum de un programa de estudios con un sentido global e internacional (Tonkin y Edwards, 1981), si bien otros autores como (Ryan, 2000; Parker y Jary, 1995) muestran preocupación por la posibilidad de que esta modalidad conduzca a un currículum uniformizado y sometido a los designios del mercado, promoviendo una visión monocultural y anglocéntrica como si fuera universal ${ }^{12}$.

\section{II-. Internacionalización en el mundo.}

El parlamento europeo realizó un estudio donde se plantea que "en Europa el proceso de IES comenzó con los programas europeos, especialmente ERASMUS, que generaron una comprensión y unos mecanismos comunes para la internacionalización. El proceso de Bolonia reforzó lo anterior. También es evidente que los programas y modelos europeos han influido en el desarrollo de la IES en otras regiones del mundo" (Parlamento europeo, 2015) ${ }^{13}$

Por otra parte, las estrategias de las universidades españolas para la internacionalización plantean "la nueva generación de programas educativos (Erasmus Plus) y de investigación

\footnotetext{
${ }^{10}$ Estrategia para la internacionalización de las universidades españolas 2015 - 2020, gobierno de Españaministerio de educación, deporte y cultura, 2014.

${ }^{11}$ Wachter, B. (2003). An introduction: Internationalisation at home in context. Journal of Studies in International Education, 7(1), 5-11.

12 Desde el sur: miradas sobre la internacionalización. - 1a ed. - Remedios de Escalada: Ediciones de la UNLa Universidad Nacional de Lanús, 2014, Buenos Aires, Argentina.

${ }^{13}$ Parlamento Europeo, 2015 Internacionalización de la Educación Superior, en http://www.europarl.europa.eu/supporting-analyses.
} 
(Horizonte 2020), en el marco de nuevas políticas de investigación y de innovación coincide con reformas en educación superior en muchos países." ${ }^{14}$.

\section{III-. Procesos de internacionalización en América Latina.}

En América latina y el Caribe "Argentina, Brasil y México han sido pioneros en el apoyo a programas de internacionalización y las indagaciones publicadas en esos tres países son numerosas y focalizadas sobre cuestiones vinculadas a la educación superior y la ciencia; aunque una producción significativa cuajó en períodos distintos (México desde los noventa, Brasil a partir del 2000, Argentina a finales de esa década) y en diferentes marcos geográficos de cooperación universitaria, los repasos bibliográficos indicaron que los investigadores atendieron un número relativamente amplio de asuntos vinculados a las políticas y programas, a la movilidad y a la internacionalización del currículum en cada uno de esos países" 15 .

Se puede apreciar en la principal motivación para la internacionalización, una visión institucional ampliamente compartida por las universidades de la región, donde se concibe la educación como parte del mundo globalizado, donde el desarrollo de competencias profesionales requiere entonces el plus formativo que adquieren los estudiantes de instituciones con una política de internacionalización explicita, como parte de su oferta académica diferenciadora.

\section{IV-. Internacionalización de la educación superior en Chile.}

En Chile, el proceso de internacionalización de la educación superior se remonta a la década de 1950, época en que algunas de las universidades más antiguas desarrollaron programas de intercambio internacional, firmando acuerdos con instituciones de otros países y permitiendo que los académicos realicen estudios de posgrado en los Estados Unidos de Norteamérica y Europa. Luego, "las reformas de la década de 1980 le otorgaron una mayor relevancia al proceso de internacionalización, ya que la introducción del modelo de mercado generó una gran competencia entre las instituciones de educación superior en búsqueda de una mayor diferenciación entre ellas"16.

En el período que va desde mediados de los setenta hasta los primeros años de los ochenta tendió a culminar hacia fines del período la formación de posgrado en el exilio y una buena cantidad de científicos sociales logró ubicarse profesionalmente en el extranjero y afianzar una carrera académica y se tendió a canalizar cada vez más recursos hacia los centros extrauniversitarios que lograban financiarse solo con recursos externos ${ }^{17}$.

Por su parte, José Joaquín Brunner plantea que para reorientar las políticas de los noventa, para el sistema en su conjunto, el Ministerio de Educación (MINEDUC) elabora y aprueba el documento "Marco de Políticas para la Educación Superior", donde en relación al objetivo de la internalización, apoyó a las instituciones por la vía de la internacionalización y la exportación de servicios universitarios e impulsó también la internacionalización de las universidades de

\footnotetext{
${ }^{14}$ Estrategia para la internacionalización de las universidades españolas 2015 - 2020, gobierno de EspañaMinisterio de educación, deporte y cultura, 2014.

15 Internacionalización de la Educación Superior y la Ciencia en América Latina: Un Estado del Arte, Sylvie Didou Aupetit \& Vielka Jaramillo de Ecobar, Caracas, 2014.

16 OCDE-Banco Mundial (2009). “La Educación Superior en Chile. Revisión de Políticas Nacionales de Educación” en

“Internacionalización de las Ciencias en América Latina; un estado del arte. Caracas, 2014.

${ }^{17}$ Garretón, Manuel Antonio (2005). Las Ciencias Sociales en Chile. Institucionalización, ruptura y renacimiento. Santiago, en “Internacionalización de las Ciencias en América Latina; un estado del arte. Caracas, 2014.
} 
investigación y, en general, de la comunidad científica, y construyó un nuevo marco de gestión y financiamiento para el fomento de la innovación ${ }^{18}$.

\subsection{Estudiantes extranjeros de intercambio en Chile.}

El 69,5\% de los estudiantes extranjeros regulares del año 2014 estaban matriculados en una universidad: $38,8 \%$ en universidades privadas, $19,8 \%$ en universidades estatales y un $10,8 \%$ en universidades privadas del Cruch. Por otra parte, un 19,2\% estaba matriculado en un instituto profesional y un $11,3 \%$ en un centro de formación técnica ${ }^{19}$.

Los principales países de los que eran ciudadanos los estudiantes extranjeros regulares en Chile el año 2014 fueron Perú (28,4\%), Colombia (19,7\%) y Ecuador (9,7\%). De los países que no pertenecen a América Latina, la mayor proporción corresponde a España $(1,1 \%)$ seguido de Francia $(0,7 \%)$, Estados Unidos $(0,6 \%)$ y China $(0,5 \%)^{20}$.

La cantidad de estudiantes extranjeros matriculados en Chile aumentó progresivamente desde al año 2014, encontrándose actualmente que "estudiantes extranjeros de intercambio matriculados en instituciones de educación superior en Chile, en 2018, es de 9.312, mostrando un aumento de 1.912 estudiantes más $(25,8 \%)$ que en el año 2014 (SIES, 2019). ${ }^{21}$

En relación a la nacionalidad de los estudiantes extranjeros de intercambio 2018, "existe una gran diversidad que alcanza los 84 países, destacando Estados Unidos (20,4\%), México $(13,8 \%)$, España $(12,4 \%)$, Francia $(11,4 \%)$, Colombia (7,5\%) y Alemania (6,5\%)". (SIES, 2019) 22

Tabla 7: Evolución de la matrícula de estudiantes extranjeros de intercambio por región mundial de origen (nacionalidad) 2014-2018

\begin{tabular}{|l|r|r|r|r|r|}
\hline Región del mundo & 2014 & 2015 & 2016 & 2017 & 2018 \\
\hline África & - & 34 & 14 & 24 & 10 \\
\hline América del Norte & 2.018 & 1.689 & 1.828 & 1.934 & 1.963 \\
\hline Asia & 137 & 125 & 179 & 197 & 222 \\
\hline Europa & 2.806 & 3.165 & 3.471 & 3.647 & 3.749 \\
\hline $\begin{array}{l}\text { Latino América yel } \\
\text { Caribe }\end{array}$ & 2.368 & 2.855 & 2.885 & 2.917 & 3.240 \\
\hline Oceanía & 71 & 67 & 65 & 66 & 47 \\
\hline Sin Información & - & 36 & 3 & 377 & 81 \\
\hline Total & 7.400 & 7.971 & 8.445 & 9.162 & 9.312 \\
\hline
\end{tabular}

Fuente: SIES (2019) Informe sobre matrícula de estudiantes extranjeros en educación superior; matrícula 2018.

En relación al tipo de institución en que se matriculan los estudiantes extranjeros, se aprecia que "las universidades privadas no Cruch las que concentran la mayor parte de la matrícula de este

\footnotetext{
18 OCDE-Banco Mundial (2009). “La Educación Superior en Chile. Revisión de Políticas Nacionales de Educación” en “Internacionalización de las Ciencias en América Latina; un estado del arte. Caracas, 2014.

19 "Situación de la matrícula de extranjeros en educación superior en Chile: datos 2014" MINEDUC.

20 "Situación de la matrícula de extranjeros en educación superior en Chile: datos 2014" MINEDUC.

${ }^{21}$ SIES (2019) Informe sobre matrícula de estudiantes extranjeros en educación superior; matrícula 2018.

22 SIES (2019) Informe sobre matrícula de estudiantes extranjeros en educación superior; matrícula 2018.
} 
tipo de alumnos extranjeros $(42,9 \%)$, seguido por las universidades privadas del Cruch $(34,6 \%)$ y las estatales del Cruch $(21,5 \%)$ (SIES, 2019). ${ }^{23}$

\section{Tabla 8: Evolución de la matrícula de estudiantes extranjeros de intercambio por tipo de institución 2014-2018}

\begin{tabular}{|c|c|c|c|c|c|}
\hline $\begin{array}{l}\text { Tipo de } \\
\text { institución }\end{array}$ & 2014 & 2015 & 2016 & 2017 & 2018 \\
\hline U. Estatal Cruch & 1.557 & 1.883 & 1.830 & 2.070 & 1.998 \\
\hline U. Privada Cruch & 2.825 & 2.721 & 3.086 & 3.044 & 3.220 \\
\hline U. Privada & 2.980 & 3.283 & 3.448 & 3.934 & 3.998 \\
\hline IP & 38 & 77 & 76 & 111 & 93 \\
\hline CFT & - & 7 & 5 & 3 & 3 \\
\hline Total & 7.400 & 7.971 & 8.445 & 9.162 & 9.312 \\
\hline
\end{tabular}

Fuente: SIES (2019) Informe sobre matrícula de estudiantes extranjeros en educación superior; matrícula 2018.

En relación a los datos presentados se puede apreciar que las preferencias de matrícula para los alumnos de intercambio se mantienen sobre las universidades privadas, quienes tienden a incluir entre sus sellos diferenciadores la internacionalización como uno de sus atractores principales en su oferta académica.

En cuanto a la distribución por sexo de los estudiantes extranjeros de intercambio, se observa una clara mayoría de mujeres, quienes, para el año 2018, representan el 60,8\% del total, participación similar a la observada en años anteriores.

\section{Tabla 9: Evolusion de la matrizula de estudiantes extranieros} de intercambio por sexo 2014-2018

\begin{tabular}{|l|r|r|r|r|r|}
\hline Sombre & 2016 & 2.215 & 2016 & 2017 & 2013 \\
\hline Mujer & 2.861 & 2974 & 3.263 & 3.506 & 3.650 \\
\hline Sin información & 4539 & 4997 & 5.173 & 5.653 & 5.660 \\
\hline Total & 0 & 0 & 4 & 3 & 2 \\
\hline
\end{tabular}

Fuente: SIES (2019) Informe sobre matrícula de estudiantes extranjeros en educación superior; matrícula 2018.

En cuanto a la distribución geográfica en relación a los alumnos de intercambio que llegan a Chile, se puede apreciar que "destacan las regiones Metropolitana (65\%) y de Valparaíso (22,7\%). La región Metropolitana, entre los años 2014 a 2018 ha tenido un crecimiento del 30\% en el número de estudiantes extranjeros de intercambio, superior al aumento observado a nivel país $(25,8 \%)$. (SIES, 2019) ${ }^{24}$

\footnotetext{
${ }^{23}$ SIES (2019) Informe sobre matrícula de estudiantes extranjeros en educación superior; matrícula 2018.

${ }^{24}$ SIES (2019) Informe sobre matrícula de estudiantes extranjeros en educación superior; matrícula 2018.
} 


\section{V-. Gestión académica.}

La internacionalización es una de las maneras con que la universidad puede diversificar y aumentar su matriz de ingresos, así como propiciar un desarrollo académico intra e inter institucional a través de alianzas estratégicas por áreas de especialización, que favorezcan la captación, retención de talentos, la producción de conocimientos, la innovación del saber y hacer.

En este sentido, desde la internacionalización pueden generar ingresos que le permitan a la universidad sustentar su gestión con grados crecientes de excelencia académica y vinculación con el medio nacional, regional e internacional.

De acuerdo a os conceptos señalados anteriormente, se pueden realizar las siguientes acciones para favorecer este proceso:

a) Potenciar la oferta académica al ofrecer una formación de pregrado con posibilidad de práctica internacional y proyecciones de posgrado fuera del país.

Esta modalidad formativa tiene un claro efecto diferenciador y atractivo para muchos jóvenes y profesionales que les interesan los valores agregados que conlleva estudiar una carrera, como el estudio de idiomas, conocer otras culturas, agregar elementos formativos diferenciadores a su currículum al momento de egresar, ampliar sus horizontes formativos más allá de las fronteras del país y aumentar considerablemente su red de contactos, todos elementos que suman a favor de aumentar la empleabilidad y entregar al mercado laboral un profesional competitivo a nivel internacional.

b) Diseño de cursos en formación continua, que se sume a la oferta académica en el área de especialización y posgrado.

Esta área es una innovación curricular que permite sumar a la diversificación de la matriz de ingresos, la posibilidad de establecer nuevas alianzas y profundización de relaciones académicas y comerciales con las instituciones en convenio, así como favorecer una proactiva vinculación con el medio, al detectar, diseñar y ejecutar cursos de especialización a medida.

Esta modalidad también es un espacio abierto a la creatividad para generar conocimiento, la innovación en la formación y la vía para realizar aportes formativos y profesionales a la sociedad, como una forma de desarrollar la responsabilidad social universitaria.

Estos cursos se pueden impartir en las siguientes modalidades:

1-. Presencial.

2-. Semi presencial.

3-. Online.

Para la segunda modalidad se puede realizar una oferta que incluye una semana presencial intensiva, con valor agregado de tours y visitas técnicas según área de especialidad, lo que por un lado es una innovación como modelo educativo y por otro agrega valor a la formación, pues incluye elementos culturales que desarrollan el concepto de internacionalización comprensiva, pues implica una formación que se abre a incluir aspectos de la cultura como enfoque institucional.

c) Diseño y desarrollo de investigaciones, publicaciones conjuntas y co-publicación, orientadas al aporte social, educativo, empresarial, científico, etc, que genere una visibilidad y presencia académica creciente de la universidad en el medio nacional e internacional. 
Esta área es relevante para el desarrollo institucional proponer un camino de avance en la generación del conocimiento que favorece las alianzas estratégicas y presencia intelectual de la universidad en el medio académico.

Las investigaciones proyectadas son de distinto tipo y área de especialidad de acuerdo a las alianzas académicas que pueden realizar las facultades dentro de los convenios institucionales.

La investigación tiene también un rol importante al perfilar las áreas de especialidad que la universidad quiere proyectar a través de sus posgrados y alianzas de estos con las universidades en convenio, manteniendo en todo momento contacto con la realidad nacional e internacional, para responder a las necesidades sociales y formativas del entorno.

En el pregrado, tienen un impacto también al interior de las escuelas, al constituirse en parte de la cultura formativa de este, mediante la creación de metodologías de aula innovadoras que involucren la investigación, a través de trabajos de campo, análisis bibliográficos, creación de paneles investigativos y ferias temáticas asociadas a las asignaturas, intra e inter universidades, que pueden organizar las escuelas semestralmente para fomentar la práctica investigativa desde este nivel formativo, sentando las bases para preparar la continuación de estudios e investigación de posgrado a nivel nacional e internacional.

d) Diseño curricular conjunto para áreas de especialización y posgrado que genere una nueva oferta académica con doble titulación y que pueda además sumar a la innovación curricular en el pre y posgrado de las universidades en convenio, lo que puede aumentar los ingresos la oferta académica conjunta internacional.

Esta área favorece una diferenciación atractiva al responder a un diagnóstico y detección de nuevas áreas para la innovación en base a las necesidades observadas en el vínculo con el medio local, nacional e internacional, tanto en los mercados laborales a donde pasan los estudiantes una vez egresados, como en los aportes sociales que la universidad debe y puede contribuir con su oferta formativa.

\section{e) Actividades de los docentes.}

Diseñar y potenciar las siguientes líneas de desarrollo de la internacionalización a este nivel:

1-. Propiciar la inclusión de metodologías que incluyan la interculturalidad en cada asignatura.

2-. Favorecer la creación de comunidades docentes a nivel internacional, especialmente con las IES

en convenio.

3-. Propiciar que los docentes incluyan textos y papers en otro idioma (de preferencia inglés).

4-. Favorecer la movilidad saliente y entrante de docentes en actividades académicas con las IES en

convenio.

f) Actividades de los alumnos.

1-. Favorecer la creación de comunidades de estudiantes a nivel internacional, especialmente con las IES en convenio.

2-. Motivar el cocimiento de conceptos, teorías y aplicaciones en otros países. 
3-. Propiciar la movilidad saliente y entrante de estudiantes con las IES en convenio.

En relación a esta mirada conceptual, las iniciativas que se pueden proyectar desde el área de internacionalización tienen un efecto diferenciador que perfila la universidad abriendo un camino de desarrollo de liderazgo en la originalidad en varias de estas, donde el énfasis está en conceptos clave como "Alianzas-Colaboración-Creatividad-Interculturalidad".

De esta manera, la internacionalización de las IES supone un concepto sistémico e integrador, donde los intercambios estudiantiles son sólo parte de un proceso más amplio, que incluye aspectos de políticas, planificación e interculturalidad para un proceso que impacte en el desarrollo institucional, para lo cual se requiere de una visión común y compartida entre cada una de las unidades de la universidad, que en definitiva es parte de un contexto cultural para proyectarse más allá de las fronteras.

\section{Referencias bibliográficas.}

CRES 2018 "Conferencia Regional de Educación Superior de América Latina y el Caribe”, Córdova, Argentina. De Wit, H. (2001). internationalisation of higher education in the United states of America and europe: A historical, comparative and conceptual analysis. Westport: Greenwood.

Gacel-Ávila, J. (2003). La internacionalización de la educación superior. Paradigma para la ciudadanía global. Guadalajara: Universidad de Guadalajara.

Gacel-Ávila, J. (2006). la dimensión internacional de las universidades. contexto, procesos, estrategias. Guadalajara, México: Universidad de Guadalajara.

Gacel Ávila Jocelyne (2014) "Proceso de Internacionalización en América Latina y el Caribe".

Gacel-Ávila, J. (2003). La internacionalización de la educación superior. Paradigma para la ciudadanía global. Guadalajara: Universidad de Guadalajara.

Gacel-Ávila, Jocelyne. (2017) "Estrategias de internacionalización de la educación superior: implementación, evaluación y rankings, México: UNESCO-IESALC).

Garretón, Manuel Antonio (2005). Las Ciencias Sociales en Chile. Institucionalización, ruptura y renacimiento. Santiago.

Gobierno de España-Ministerio de educación, deporte y cultura, (2014) "Estrategia para la internacionalización de las universidades españolas 2015 - 2020".

Hudzik, J. K. (2011). comprehensive internationalization: from concept to action. Washington: nafsa.

OCDE-Banco Mundial (2009). “La Educación Superior en Chile. Revisión de Políticas Nacionales de Educación”.

Parlamento Europeo, 2015 Internacionalización de la Educación Superior, en http://www.europarl.europa.eu/supporting-analyses.

Rudzki, R. (1998). "The strategic management of internationalization-Towards a model of theory and practice". Tesis Doctoral, Newcastle, School of Education, University of Newcastle upon Tyne.

SIES (2019) Informe sobre matrícula de estudiantes extranjeros en educación superior; matrícula 2018.

Sylvie Didou Aupetit \& Vielka Jaramillo de Ecobar, Caracas, (2014), "Internacionalización de la Educación Superior y la Ciencia en América Latina: Un Estado del Arte".

Tangelson, Guillermo (2014) "Desde el sur: miradas sobre la internacionalización”. - 1a ed. - Remedios de Escalada: Ediciones de la UNLA - Universidad Nacional de Lanús, 2014, Buenos Aires, Argentina.

UNESCO (2013). Global Citizenship Education: An Emerging Perspective.

Wachter, B. (2003). An introduction: Internationalization at Home in context. Journal of Studies in International Education 7 (1): 5-11. 